\title{
Underspecified changes: a dynamic, probabilistic frame theory for verbs ${ }^{1}$
}

\author{
Ralf NAUMANN — Heinrich Heine University Düsseldorf \\ Wiebke PETERSEN — Heinrich Heine University Düsseldorf \\ Thomas GAMERSCHLAG — Heinrich Heine University Düsseldorf
}

\begin{abstract}
The verb 'rise' can be used both with property-denoting nouns like 'temperature' but also with NPs like 'a Titan' or 'China'. Whereas in the former case the change triggered by a rising event is directly related to the subject (its current value increases), this does not hold for 'a titan' or 'China'. In this case it is a property of these objects, say their height or their political power, which increases in value. Furthermore, 'rise' does not target a particular property as the examples above show. This data has led Cooper (2010) to the conclusion that it is presumably not possible (i) "to extract a single general meaning of words which covers all the particular meanings of the word in context", and (ii) "to determine once and for all the set of particular contextually determined meanings of a word". In this article we present a solution to the two problems raised by 'rise' in a frame theory. 'Rise' is analyzed as a scalar verb which does not lexicalize a complete scale in its meaning. Rather, it shows underspecification relative to the dimension (property) parameter of a scale. The set of admissible properties is determined by a constraint on the value ranges of properties. If the property is not uniquely determined by the subject, the comprehender uses probabilistic reasoning based on world knowledge and discourse information to defeasibly infer the most likely candidates from this set (2nd problem). The first problem is solved not by simply introducing objects into the representation of a discourse but instead by pairs consisting of an object and an associated frame component which collects the object information contributed by the discourse. Changes triggered by events like the one denoted by 'rise' are modelled as update operations on the frame component while the object component is left unchanged.
\end{abstract}

Keywords: lexical semantics, scalar changes, frame theory, probabilities.

\section{Two puzzles about 'rise'}

According to Cooper (2010), the question 'What is the meaning of an item?' is divided into the following two subquestions: (i) "is it possible to extract a single general meaning of words which covers all the particular meanings of the word in context?", and (ii) "is it possible to determine once and for all the set of particular contextually determined meanings?" For Cooper, data like that in (1) shows that the answer to both questions is most likely 'no'. (Examples (1c) - (1e) taken from Cooper 2010.)

(1) a. The temperature (of the liquid) is rising.

b. The price (of the commodity) is rising.

c. As they get to deck, they see the Inquisitor, calling out to a Titan in the seas. The giant Titan rises through the waves, shrieking at the Inquisitor.

d. Mastercard rises.

e. China rises.

${ }^{1}$ The research was supported by the German Science Foundation (DFG) funding the Collaborative Research Center 991. We would like to thank the reviewers and audience of SuB22 for helpful comments and suggestions. 
In order to arrive at a single general meaning for 'rise', it is necessary to characterize the contribution of the subject argument in a unique way. However, the data in (1) shows most likely that this cannot be done. In (1a) and (1b) the change expressed by 'rise' is directly related to the denotation of the subject argument. It is the value of the temperature/price that increases. This does not hold for the remaining three examples. The referent of 'titan', 'Mastercard' and 'China' must be held constant. What changes, rather, is the value of a property of the subject referent. For example, in (1c) it is the height of the titan that increases. Hence, there are conflicting constraints imposed by the subjects in (1a) and (1b) on the one hand and the other three examples on the other hand. Even if one focuses on the property that gets changed, there is no uniformity because different properties such as temperature, price and height are involved in these examples. Cooper concludes: 'This makes it difficult to see how we could give a single type which is general enough to include both varieties and still be specific enough to characterize the meaning of rise' (Cooper, 2010).

The 'Mastercard' and 'China' examples are taken by Cooper as evidence that the second question has to be answered in the negative too. Cooper comments: "While speakers of English can get an idea of the content of the examples in (1d) and (1e) when stripped from their context, they can only guess at what the exact content might be. It feels like a pretty creative process" (Cooper, 2010). The problem stems from the fact that given an object like 'China' or 'Mastercard' there are in general many properties that can be targeted by 'rise'. What is required, therefore, is an explicit context like the one given by Cooper for 'China rises' in which particular properties are singled out:

(2) "The rise of China will undoubtedly be one of the great dramas of the twenty-first century. China's extraordinary economic growth and active diplomacy are already transforming East Asia, and future decades will see even greater increases in Chinese power and influence. But exactly how this drama will play out is an open question. Will China overthrow the existing order or become a part of it? And what, if anything, can the United States do to maintain its position as China rises?"

Cooper's argument can be summarized in the following way: (i) 'rise' semantically targets a property whose value is increased by an event of this sort; (ii) this property is not uniquely determined; (iii) there seems to be no principled way to characterize or define the set of admissible properties once and for all so that for any given context one element of this set is selected by 'rise' (second problem); and (iv) the subject argument of 'rise' either denotes the targeted property or an entity which has this property. In the latter but not in the former case, the denotation of the subject argument is held constant (first problem).

In this article we will propose a solution to these two puzzles in a frame theory. In contrast to Cooper's variant of such a theory which is based on records we apply a variant of frame theory outlined in (Petersen, 2007) and elaborated on for dynamic frames in Naumann (2013) and Gamerschlag et al. (2014). We incorporate insights from two research traditions: (i) 'temperature' and 'price' are (basically) functional nouns, i.e. they semantically relate a property with an object that has this property and a value of this property. This semantic representation closely resembles the frame representation of nouns in general in terms of typed attribute value 
structures. Hence, in our frame theory, all nouns are represented in terms of frames. Furthermore, discourse objects are pairs consisting of an object and an associated frame component. Changes, and more generally updates, are always executed on the frame component while the object component is held constant. This is the key to the solution of the first puzzle. (ii) The key observation for a solution of the second puzzle comes from the notion of a scale. Each admissible attribute is associated with a particular scale which specifies a dimension, a set of values, and an ordering relation on them. 'Rise' shows attribute-underspecification: no particular dimension is specified. Rather, it imposes a constraint on the ordering relation defined for the attribute values. Only those properties (dimensions) are admissible which satisfy this constraint.

Before presenting our analysis of 'rise', we will first introduce some theoretical prerequisites in the following sections which deal with functional concepts, scalar changes and their relation to frames.

\section{Inherently functional nouns with particular value ranges}

One way of analyzing nouns like 'temperature' and 'price' is as being basically inherently functional, i.e. as denoting a functional concept, cf. Löbner (2011). ${ }^{2}$ For example, the temperature is always the temperature of something else. Possible objects are persons (Bill's temperature), bodies (the temperature of the sphere) and three-dimensional spatial regions (the ambient temperature). Similar observations apply to 'price'. The two objects targeted by such nouns are linked in a particular way. The object denoted by 'the $\mathrm{N}$ ' in an identity statement like 'The temperature is 90 degrees Celsius' or 'The price is 30 Euro' is the value of a property of the object left implicit in those statements. Making the relation explicit yields for example 'The ambient temperature is 90 degrees Celsius'. Here, the noun denotes the property itself, e.g. 'temperature'. Let us call the value of the property the 'dependent object' and the object the value is related to by the property the 'independent object'. For example for 'The temperature of the liquid is 90 degrees Celsius', one gets: temperature $\doteq$ property, $90 \doteq$ dependent value and the liquid $\doteq$ independent object. Two properties of functional nouns like 'temperature' and 'price' are (i) they are time-dependent and (ii) for a given independent object and time point there is a unique value, i.e. dependent value. Examples of inherently functional nouns that are also time-dependent and (almost) functional are 'president' and 'husband'. The difference between 'temperature' and 'price' on the one hand and 'president' and 'husband' on the other lies in properties of the set of (dependent) values. Whereas for the former this set is linearly ordered (100 degrees Celsius is greater than 50 degrees Celsius) there is no ordering on the set of possible presidents or the set of possible husbands. This difference shows up in the admissibility of 'rise'.

a. The temperature is rising/changing.

b. The president is *rising/changing.

The distinction between dependent and independent objects is directly related to one of the

\footnotetext{
2 'Basically' means that there are other uses as well. For example, 'temperature' can be used as a sortal noun, see Löbner (2011) for details.
} 
problems discussed in the first section. The subject of 'rise' denotes either a property of an object the value of which is changed by an event of rising or the object the value of a property of which is changed by a rising event. Hence, if the subject does not denote a property, it denotes an entity a property of which is targeted by 'rise'. The task, therefore, is to find a representation of nouns in which they are not simply interpreted as sets of objects or as sets of pairs of objects but in which also their properties become available.

\section{Scalar changes and scalar underspecification}

Rappaport Hovav and Levin (2010) make a basic distinction in the verbal domain between result verbs and manner verbs. Examples of both types of verbs are given in (4).

(4) result verbs: clean, cover, empty, fill, freeze, kill, melt, open, arrive, die, enter, ... manner verbs: nibble, rub, scribble, sweep, flutter, laugh, run, swim, ...

These two classes of verbs differ with regard to what they lexically encode as part of their meaning. Result verbs encode scalar changes whereas manner verbs encode non-scalar changes. According to Rappaport Hovav and Levin (2010: p.8), a scalar change in an entity involves a change in value of a particular dimension for this entity in a particular direction along the scale, with the direction specified by the ordering relation. Hence, scalar changes are changes in the value of a single property or attribute of an entity. By contrast, non-scalar changes cannot be characterized in terms of an ordered set of values of a single attribute (Rappaport Hovav and Levin, 2010: p.12). Rather, they involve complex changes - that is, a combination of multiple changes - and this complexity means that there is no single, privileged scale of change (Rappaport Hovav and Levin, 2010: p.12). Scalar changes are based on the notion of a scale, "where a scale is a set of degrees - points or intervals indicating measurement values-on a particular dimension (e.g., height, temperature, cost), with an associated ordering relation" and "verbs denoting events of scalar change lexically specify a scale", (Rappaport Hovav and Levin, 2010: p.8). According to Kennedy and McNally (2005), a possible definition of this notion is: a scale is a triple $\langle S, \Delta, R\rangle$ s.t. $S$ is a set of degrees, $\Delta$ is the dimension of measurement and $R$ is an ordering on $S$. Thus, following Rappaport Hovav and Levin (2010) different scalar verbs encode different scales, some of them only differing by the order of degree-points. By way of example, consider the two verbs 'cool' and 'warm', which lexicalize a property scale. For both verbs, the dimension of measurement is that of the temperature of an entity and the values are therefore temperature degrees. They differ w.r.t. to the direction along the temperature scale. Whereas for 'warm' there is a change from a smaller to a greater temperature degree (increase in temperature) for 'cool', this direction is reversed: the temperature decreases.

Rappaport Hovav and Levin (2010) argue that change of state verbs always lexicalize a complete scale in the sense that all three parameters are specified in the lexicon. If a verb does not lexicalize a complete scale, it does not encode a scalar change according to the authors. For example, Rappaport Hovav and Levin (2010) do not classify 'cross' and 'traverse' as verbs encoding a scalar change. Though these verbs lexically specify a dimension of measurement (a path) and, therefore, a set of values (position on the path), the direction of motion along this path is not lexically specified and, hence, they do not impose an ordering on the points of the 
path. The view that scalar changes require a complete scale as part of their lexical meaning has been challenged in Fleischhauer and Gamerschlag (2014), who show that change of state verbs coming with a property scale do not necessarily encode a complete scale but can also exhibit scalar underspecification. For instance, none of the verbs in (5) specifies any particular dimension in its lexical meaning that is targeted and depends upon the functional noun realized in subject position to introduce a dimension such as temperature, price and pressure. The verbs 'rise' and 'fall' in the first two examples operate on the scale provided by the subject and express the 'direction of change', i.e. whether the change results in an increase or decrease of values. The verb 'change' in the third example is even less specific since it refers to a change along the dimension expressed by the subject without encoding a fixed direction of change.
a. The temperature/price/pressure is rising. $\rightarrow$ increase in temperature/price/pressure $(>)$
b. The temperature/price/pressure is falling. $\rightarrow$ decrease in temperature/price/pressure $(<)$
c. The temperature/price/pressure is changing. $\rightarrow$ increase or decrease in tempera- ture/price/pressure $(>\cup<)$

In spite of being incomplete in regard to the dimension, all of the three verbs in the example above can be characterized as change of state verbs since they already address some aspect of change,namely its direction or the fact that a change takes place at all. As will be shown in the next section, this kind of scalar underspecification is the key to our solution of the second puzzle, i.e, the question how the context determines the meaning of a lexical item.

\section{4. (Scalar) changes in a frame theory}

Frame theory is based on the notion of an attribute. Attributes are interpreted as functional binary relations. In addition to being functional they are typed (or sorted). For each attribute, there is a source sort and a target sort. For example the attribute COLOR has the source sort physical object and the target sort color. For TEMPERATURE, the source sort is physical object too but the target sort is temperature. A sort $s$ is interpreted as a subset $D_{s}$ of the global domain $D$. If $s$ is the target sort of an attribute, $D_{s}$ will be called the value range of this attribute. One way of classifying attributes is in terms of instances (or subtypes) of more general relations. Löbner (2014) distinguishes four classes: (i) mereological attributes (HEAD, HANDLE), (ii) role/correlate attributes (PRESIDENT, SPOUSE), (iii) property attributes (WEIGHT, TASTE) and (iv) event-related/affordance attributes (PURPOSE). The value ranges of attributes can be classified according to their ordering properties. A basic distinction is that between 'unordered', denoted by $\perp$, and 'ordered', $<$. If the set is ordered, relevant properties are 'linear' $\left(<_{\text {linear }}\right)$, 'existence of a minimal element' $\left(<_{\min }\right)$ and 'existence of a maximal element' $\left(<_{\max }\right)$. An attribute dimension is a triple $\left\langle\Delta_{\mathrm{ATTR}}, D_{s}\right.$, ord $\rangle$ s.t. $\Delta_{\mathrm{ATTR}}$ is the interpretation of ATTR, $D_{s}$ is the value range of ATTR and ord is the ordering defined on this value range. In the present context ord is always at least linear. However, particular verbs can impose stronger conditions, e.g. that the linear order has a minimal but no maximal element. As will be shown below in section 6 this is the case for 'rise'. Non-stative (or dynamic) verbs operate on the set of attribute dimensions in the frame components of their arguments. They (possibly) im- 


\begin{tabular}{l|c|c|c} 
verb & $\begin{array}{c}\text { unique attribute } \\
\text { specified }\end{array}$ & $\begin{array}{c}\text { linear order } \\
\text { required }\end{array}$ & $\begin{array}{c}\text { direction of } \\
\text { change }\end{array}$ \\
\hline cool, widen & + & + & + \\
\hline rise & - & + & + \\
\hline cross & + & + & - \\
\hline rename & + & - & - \\
\hline change & - & - & - \\
\hline$? ?$ & - & + & - \\
\hline non existent & - & - & + \\
\hline non existent & + & - & + \\
\hline
\end{tabular}

Table 1: Classification of change of state verbs

pose constraints on the following three parameters: (i) a particular attribute is specified (yes: + / no: -); (ii) 'ord' is required to be (at least) linear (yes: + / no: -) and (iii) a direction of change is specified or not (yes: $+/$ no:-). Table 1 shows the eight possible combinations of these three parameters. The direction of change $\vec{R}$ is defined in terms of ord: if ord $=\perp, \vec{R}=\perp$ and if ord $=<_{\text {linear }}, \vec{R} \in\left\{<_{\text {linear }},>_{\text {linear }},><_{\text {linear }}\right\}$. Here, we use the following notation: (i) $\leq:=<\cup$ id; (ii) $>:=\leq^{c}$ and (iii) $><:=>\cup<$.

A verb expresses a scalar change if the value range is required to be linearly ordered and the direction of change $\vec{R}$ is either increasing or decreasing. In contrast to Rappaport Hovav and Levin (2010), we neither claim that a verb specifying a scalar change necessarily determines the attribute dimension nor that the direction of change is always fixed; thus our approach is in line with the definition of a scalar change in Fleischhauer and Gamerschlag (2014). If a verb does not require $D_{s}$ to be ordered though it is compatible with such an ordering, we set $\vec{R}$ to '-'. Hence, if ord $=<_{\text {linear }}$, a '-' amounts to $\vec{R}=><_{\text {linear }}$. This explains the compatibility of 'change' both with ordered and unordered value ranges, witness the examples in (6).

(6) a. The temperature is changing. $(\vec{R}=><$ linear $)$

b. The colour of the leaf is changing. $(\vec{R}=\perp)$

c. The president is changing. $(\vec{R}=\perp)$

Table 1 excludes combinations requiring no linear order on the value range while at the same time entailing a direction of change because the direction of change $\vec{R}$ is defined in terms of the ordering on the value range. Note that the combination 'unique attribute specified= -', 'linear order required $=+$ ' and 'direction of change $=-$ ' is marked by question marks in the table above since we leave it as an open question whether this particular combination is attested for a verb or not. Possible candidates might be 'level off' and 'reach' as in 'Inflation leveled off' and 'The temperature reached 30 degrees Celsius'.

\section{Attribute-underspecification}

From the three constraints on parameters in Table 1, the first two are directly related to properties of an argument that is targeted by a verb. It is only the constraint on the direction of change 
that is partly independent of properties of the target argument. ${ }^{3}$ Furthermore, if a verb lexically specifies a particular property attribute, the ordering relation is determined too because it is a property of the attribute's value range which is given by the target sort of the attribute. By contrast, imposing a constraint on the ordering does not uniquely determine a particular property attribute because different such attributes can have ordering relations on the value range with the same properties. This yields attribute-underspecification. Therefore there is an asymmetry depending on whether a unique attribute is determined or whether the properties of the ordering on the value range are specified. In the first case no further underspecification results whereas in the second case one gets attribute underspecification. However, attribute underspecification does not mean that the set of admissible (property) attributes cannot be determined once and for all. It is the set of all property attributes satisfying the constraint imposed by the ordering that are defined for the frame component of the argument targeted by the verb. If the attribute is not specified in the lexicon, it can be (uniquely) determined by the sort of the argument which is targeted by the verb. For example for 'rise', the attribute-underspecification is eliminated if the subject argument denotes an attribute whose value range is linearly ordered. This holds for 'temperature' and 'price'. If the subject argument is not an attribute-denoting noun, the underspecification is in general not eliminated because the object denoted by the subject will in general have more than one (property) attribute which satisfies the condition on its value range. ${ }^{4}$

The relation between scalar changes in our frame theory and the notion of a scale defined above in section 3 is the following. The measurement dimension $\Delta$ corresponds to a single attribute (e.g., SIZE, PRIZE, TEMPERATURE). The set $S$ is the value range of the attribute $D_{s}$, if it is specified in the lexicon. Since the parameter $\vec{R}$ is determined by the verb, there is no difference w.r.t. this parameter. The notion of an attribute is, however, more general than that of a dimension in a scale. Attributes can be used to represent arbitrary properties of objects, witness the examples above of various classes of attributes. Attributes that correspond to dimensions are property attributes with a value range that is at least linearly ordered.

\section{The constraint on the value range imposed by 'rise'}

In our frame theory verbs can impose constraints both on an attribute and the value ranges of attributes. ${ }^{5}$ 'Rise' is an example of a verb which imposes a constraint on the value range but does not specify any particular attribute in the lexicon. Hence, attribute-underspecification applies to it. The constraint on the value range is given below in (7).
a. The ordering $<$ is linear.
b. $\quad<$ has a minimal element: $\exists \delta . \forall \delta^{\prime}: \delta \leq \delta^{\prime}$.
c. $\quad<$ has no maximal element: $\forall \delta \cdot \exists \delta^{\prime}: \delta<\delta^{\prime}$ :

Hence, the ordering is bounded to the left but unbounded to the right. The third constraint is

\footnotetext{
${ }^{3}$ Remember the dependency between the parameters 'direction of change' and 'linear order required': if 'direction of change $=+$ ', then 'linear order required $=+$ '.

${ }^{4}$ If the value range of an attribute is scalar, then it is a property attribute. However, it does not hold that if an attribute is a property attribute, then its value range is scalar, witness the example of TASTE.

${ }^{5}$ In addition, verbs can impose a constraint on the source sort or target sort of an attribute.
} 
due to the empirical observation that 'rise' by itself does not impose a bound on the change. Rather, a (culminating) value can be arbitrarily fixed by a measure phrase, e.g. 'rise (by) 30m'. Examples of (property) attributes which satisfy this constraint include TEMPERATURE, PRICE, ECONOMIC_POWER and POLITICAL_POWER. Which attributes satisfy this constraint for objects of a given sort depends on the set of attributes defined for the frames of that sort. For example, of the above four examples only ECONOMIC_POWER and POLITICAL_POWER are defined for frames of sort country. ${ }^{6}$ We are now ready to define the lexical constraint imposed by 'rise'.

'rise' lexically imposes the following two constraints: (i) the subject argument has at least one attribute which satisfies the constraint on the value range given in (7) and (ii) for at least one of those attributes there is an increase relative to the value of this attribute triggered by the rising.

Though 'rise' does not single out a unique attribute, a unique set of attributes is determined, namely all those attributes whose value ranges satisfy (7). Hence, instead of specifying a set of admissible attributes in terms of one property that is common to all of them, the set of admissible attributes is determined by one property of their value ranges. This is our answer to the second puzzle of how the context determines word meaning. Before turning to our answer to the first puzzle of how to arrive at a generalized meaning representation of a lexical item, we need to introduce some more details of our frame theory.

\section{Modelling world knowledge and discourse information}

\subsection{Modelling world knowledge}

Our frame theory is based on models $\mathscr{M}=\left\langle W, D_{o}, D_{f}, D_{t}, P, R, I\right\rangle$ s.t. (i) $W$ is a finite set of worlds which are used to represent epistemic uncertainty; (ii) the domain $D_{o}=\bigcup_{\sigma \in \Sigma} D_{\sigma}$ is the union of finite domains $D_{\sigma}$ based on a partially ordered sort hierarchy $\left\langle\Sigma, \sqsubseteq_{\Sigma}\right\rangle$ with basic sorts like 'event' $(e)$ or 'individual' $(d)$; (iii) $D_{f}$ is the domain of frames. Each frame is of a sort $\sigma$ and is related to a particular world $w$ by a function $I N$ : $I N(f)$ is the world relative to which $f$ contains information about its root (details below); (iv) $D_{t}$ is a linearly ordered set of time points; (v) $P$ is a probability distribution on subsets of $W$. One has $P(\{w\})>0$ for all $w \in W$; (vi) $R$ is an accessibility relation on $W$ which is assumed to be the universal relation, i.e. $R=W \times W$; and (vii) $I$ is an interpretation function.

In our frame theory a distinction is made between objects, i.e. individuals and events, on the one side and frames on the other side. Both are atomic entities that are elements of two separate domains $D_{o}$ and $D_{f}$, respectively. Relative to a possible world and a time point each object is assigned a set of frames which is partially ordered based on the information contained in a frame belonging to this set. In a discourse, an object is associated with a frame that collects sortal and relational information about it got in the discourse (see below for details). Objects

\footnotetext{
${ }^{6}$ Note that we are aware of the fact that the exclusion of TEMPERATURE in the frame of 'China' is an oversimplification since an attribute such as AVERAGE TEMPERATURE IN SUMMER should certainly be part of this frame. However, for the sake of simplicity we exclude TEMPERATURE as an attribute interpreted as the temperature of simple physical objects.
} 
are related to frames by a function root, which maps a frame to the object which is the referent of the frame in the sense that the information contained in this frame is about this object. Each frame is of a particular sort, say person or temperature. Frames with the same root are ordered by a (partial) information ordering. This ordering is defined in terms of a function $\theta$, which assigns to each frame the set of relations (chains of attributes) plus the corresponding sortal information defined for it. A frame $f$ about an object $o$ (i.e. $\operatorname{root}(f)=o$ ) contains more information than a frame $f^{\prime}$ with the same root if $\theta\left(f^{\prime}\right) \subseteq \theta(f)$ and $\forall o^{\prime}$. $\llbracket \pi \rrbracket\left(f^{\prime}\right)(o)\left(o^{\prime}\right) \rightarrow$ $\llbracket \pi \rrbracket(f)(o)\left(o^{\prime}\right)$ for $\pi$ a chain of attributes in $\theta\left(f^{\prime}\right)$, i.e. $f$ contains all information about $o$ that $f^{\prime}$ contains and possibly some more information. Note that, (chains of) attributes are not interpreted as binary relations on $D_{o}$, but their interpretation is relativized in two respects. The first is directly linked to the fact that information about an object is always related to a particular frame. This dependency is achieved by interpreting (chains of) attributes as ternary

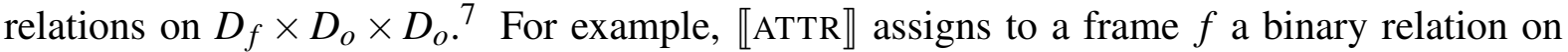
$D_{o}$ s.t. $\llbracket \operatorname{ATTR} \rrbracket(f)(o)\left(o^{\prime}\right)$ is true if $o$ and $o^{\prime}$ are related by ATTR in $f$. This way of relativizing the interpretation of expressions is similar to the way information is made world-dependent in two-sorted type theory. However, this move does not account for the fact that information in frames is in general time-dependent. The values of attributes in a frame can be changed by an event (of the appropriate sort) resulting in another frame which reflects this change of value. There are various ways of how this time-dependency can be modelled. Let $D_{t}$ be a domain of time points that is linearly ordered by $<_{t}$. One way is to use a special constant Hold on $D_{f} \times D_{t}$ s.t. Hold $(f, t)$ is true if the information contained in $f$ is true at $t$. An alternative is to relativize the interpretations of relational expressions a second time. Instead of having relations

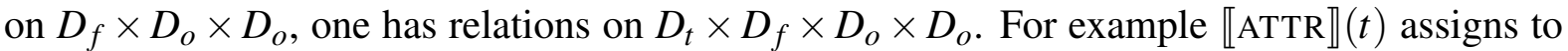
a frame $f$ a binary relation on $D_{o}$ at time point $t$. The disadvantage of the second alternative is that it makes it more difficult to compare the information contained in two frames with the same root since this information is always time-dependent.

\subsection{Possibilities, information states and discourse objects}

Besides world knowledge, discourse information has to be modelled as well. A possibility $p$ is a pair $\langle c, w\rangle$. The first component of a possibility is a stack $c$, i.e. a function from a finite initial segment of $\mathbb{N}$ to discourse objects, see below and e.g. van Eijck (2007) and Naumann and Petersen (2017) for details. The second component of a possibility is a possible world $w$. The stack $c$ is called the discourse component and $w$ the world component of the possibility. An information state is a set of possibilities together with a probability distribution $\operatorname{Pr}$ that is derived from $P$ (see below for details). Modelling information states as sets of possibilities in the way defined above accounts for epistemic uncertainty. Attribute-underspecification gives rise to such uncertainty if no particular attribute is specified, as in the case of 'China rises'. Each possibility models a possible resolution in the sense that there is (at least) one attribute which satisfies the condition on the value range in (7). For example, for China there is a possibility in which its political power increases and a possibility in which its economic power increases.

\footnotetext{
${ }^{7}$ In the context of our argument, the sort hierarchy plays a minor role. Thus, in the current paper, we mainly ignore the sortal restrictions expressed by frames. However, in parallel to the relational information expressed by attributes, the sortal restrictions expressed by sorts are interpreted not as unary relations on $D_{o}$ but as binary relations on $D_{f} \times D_{o}$.
} 
In standard semantic theories, a domain extension operation (associated with the meaning of indefinites, or more generally, quantified NPs and verbs) introduces a new object, an individual or an event, into the discourse. In our frame theory a domain extension operation is more complex. Instead of an object a pair consisting of an object and an associated frame is introduced. The frame contains the information gathered about the object plus (possibly) information based on world knowledge of a comprehender. Such pairs are called discourse objects. The first projection (component) is called the object component and the second projection the frame component of the discourse object. Getting more information triggers an update operation on the frame component of a discourse object while keeping the object component constant. Two kinds of updating operations have to be distinguished: (i) getting more 'static' information about an object, e.g. sortal and/or relational information that is not time-dependent (getting information about a static world), and (ii) getting information that is dynamic in nature, e.g. information about a property the value of which has been changed due to the object having been involved in an event (information about an evolving, changing world). Though this modelling of discourse objects works fine for non-relational nouns like 'person' or 'China', it is insufficient for functional nouns like 'temperature' 'price' or 'president'. Consider e.g. a basic frame for 'temperature'.

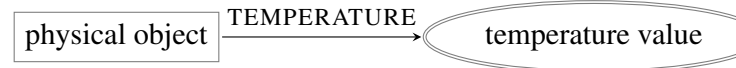

Figure 1: 'temperature' frame

In the 'temperature' frame the central node is not the root of the frame graph. Rather it has an incoming arc labeled TEMPERATURE indicating that the referent of the central node is functionally dependent on the referent of the open argument node. If this argument is filled with something of type physical object, the actual referent of the concept is determined (each physical object has a unique temperature). Thus, the central node is closed because it is determined by the open argument node. ${ }^{8}$

In order to adequately integrate functional nouns in our discourse model, we propose to model the frame component of functional nouns as a pair of frames, one frame representing the dependent object and the other frame for the independent object. ${ }^{9}$ For non-relational nouns like 'person' or 'China', the dependent and the independent frame component are the same. For functional nouns, the value of the function $\theta$ contains at least one element of the form $\otimes$ ATTR, i.e. the converse of ATTR. For example, in the case of 'temperature' one has $\theta(f)=$ $\{\otimes$ TEMPERATURE $\}$. By contrast, for non-relational (sortal) nouns no such elements exist. ${ }^{10}$ As a result, a discourse object is a pair $\left\langle o,\left\langle f_{d}, f_{i}\right\rangle\right\rangle$ consisting of the object component $o$ and the frame component $\left\langle f_{d}, f_{i}\right\rangle$, which in turn consists of the dependent frame $f_{d}$ and the independent frame $f_{i}$.

\footnotetext{
${ }^{8}$ In our frame graphs, the central node is marked by a double line; argument nodes are open nodes and denoted as rectangles while closed nodes are marked by ellipses. For more information on frame graphs see Petersen (2007) and Petersen and Osswald (2014).

${ }^{9}$ Non-functional nouns like 'sister' do not fall into the scope of the present paper; in principle, they are handled similar to functional nouns.

${ }^{10}$ For the sake of simplicity, we claim that frame graphs do not contain circles.
} 


\section{Representing changes}

Since discourse objects are pairs $\left\langle o,\left\langle f_{d}, f_{i}\right\rangle\right\rangle$, a dynamic verb can operate on all three components, at least theoretically. In our frame theory, a verb always operates on the frame component while the object component is held constant. With respect to the frame component, it is important to distinguish between constraints on a frame component and the actual change effected relative to a frame component. If the verb imposes a constraint, e.g. on the ordering of the value range of an attribute, this constraint has to be satisfied for a sort in the dependent frame and not in the independent frame. This becomes evident if one considers a functional noun like 'temperature'. If it is the temperature of a person, the person frame will in general have several property attributes whose value ranges satisfy the constraint imposed by 'rise'. However, this is not what is required. Rather, it is the value range of the temperature attribute that has to satisfy the constraint. By contrast, the change itself is modelled as an update operation on the independent frame component. This becomes again evident if one looks at an example involving 'temperature', like 'John's temperature is rising'. The dependent object is a (temperature) value (or degree). This values does not change, witness Partee's famous puzzle: 'The temperature is rising. The temperature is $90 . \sharp 90$ is rising'. What is changing (evolution of the world, ontic change) is John's temperature. Its value is higher at the end of the rising compared to its value at the beginning of the event. Hence, the relation between the components of a discourse object and the change expressed by a dynamic verb is as follows.

- The object component, e.g. a temperature value or China, remains constant.

- Any constraints must be satisfied in the dependent frame component.

- The change is defined as an update operation on the independent frame component.

Since for non-relational nouns like 'China' the two frame components are the same, the constraints and the change are both related to this frame. We are now ready to formulate our solution to the first puzzle: (i) changes are uniformly represented at the level of frames, and (ii) the objects themselves are held constant. (i) and (ii) apply to all arguments, in particular to relational (functional) and non-relational nouns, alike.

\section{Static and dynamic frames}

A basic frame for 'rise' has a single attribute THEME. At this level the rising event, i.e. the value of $\operatorname{root}\left(f_{\text {rise }}\right)$, is taken as an atom. By a zooming operation $Z$ in the sense of Blackburn and deRijke (1997) this atomic event is decomposed into single step subevents. This level is called the 'event decomposition' level (ED). It represents the temporal structure of the event and links it to the level of the described situation, the participating objects, and their roles in the event. Each temporally extended event $e$ on the ED level is bounded by two boundary events $\alpha(e)$ (left boundary) and $\beta(e)$ (right boundary) whose runtimes are singletons (cf. Pinon, 1997). Non-boundary events are linked to global properties of the event, termed 'static event frames' (SEF) in Naumann (2013). The basic frame for 'rise' described above is of this sort. Boundary events are linked to situation frames (SF) which are built up from the frames for the objects involved. The SFs specify the relevant information about the attribute involved in the change, e.g. HEIGHT or TEMPERATURE. Let us illustrate this with 'The balloon rises by $30 \mathrm{~m}$ '. 


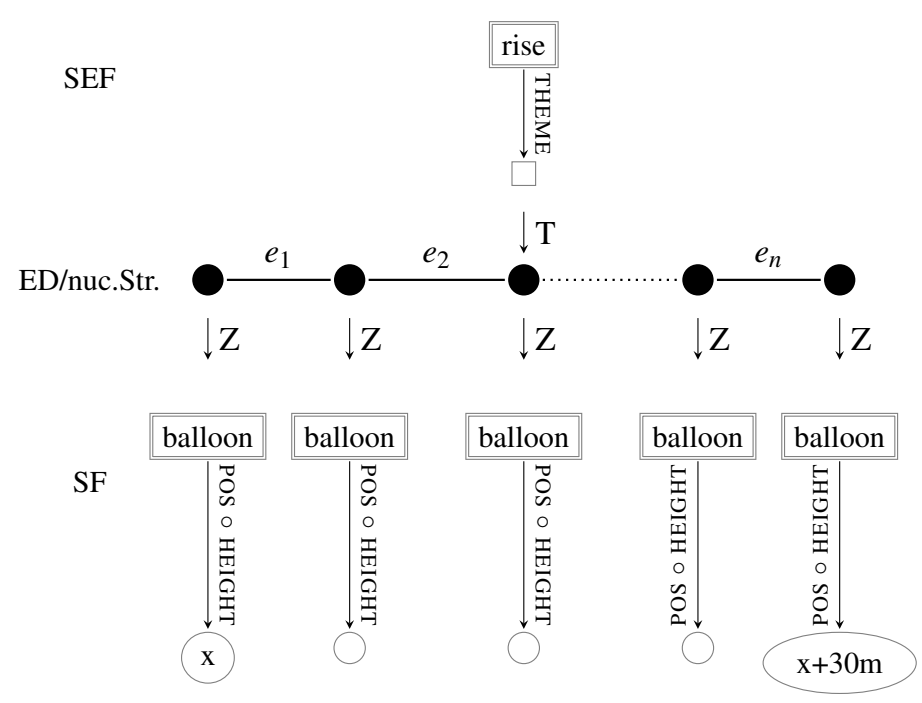

Figure 2: dynamic frame for 'The balloon rises by 30 meters'

At the top the basic SEF for 'rise' with the single THEME-attribute is shown. The ED-level is shown below. Formally, it is achieved by a zooming operation of type 'temporalization' (T). At this level the rising event is decomposed into a sequence $e_{1} \cdots e_{n}$ of atomic rising events whose sum is just the rising event which is the root of the rising frame. Each $e_{i}$ is bounded by two boundary events, represented by the black bullets. As said above, each boundary event is related to a particular time point. The SF at the timepoint of each boundary event gives the information about the attribute whose value gets changed. In this case it is the value of the chain POSITION $~$ HEIGHT in the frame of the balloon. The SF level in the figure provides snapshots of the balloon's height at different time points of the event. A condensed representation in a single frame is given below. Note that 'trace' and 'change' are "dynamic attributes" which are projected into this frame from the event decomposition frame introduced above. Attributes of this type have the function to record the value change of attributes such as POSITION and HEIGHT over the course of the event (for details see Gamerschlag et al., 2014).

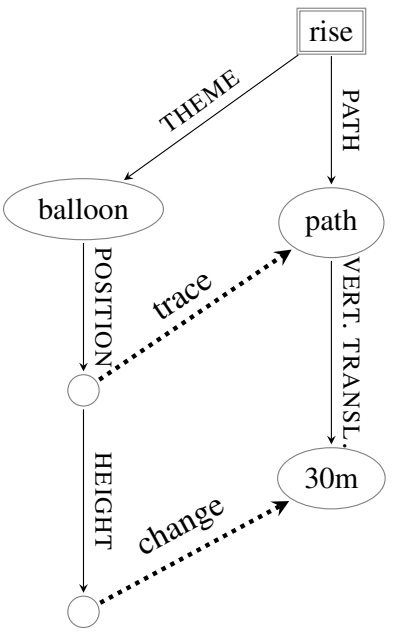

Figure 3: condensed dynamic frame for 'The balloon rises by 30 meters' 


\section{The dynamic meaning of 'rise'}

The interpretation of 'rise' triggers both a domain extension operation and an update operation. First, an event of rising is introduced into the discourse. Second, the domain object related to the theme argument, e.g. 'China', is updated. This update operation is related to the change triggered by a rising event. For scalar verbs in general, the change is effected relative to an attribute of some argument (as discussed in section 4). If the verb shows attribute-underspecification, no particular attribute is singled out. In this case the current information state must be updated for each attribute that satisfies the constraint imposed by 'rise' on the value range. This has the effect that for a possibility $p_{i}$ in the input, there can be more than one successor possibility in the output. In particular, one has: for each possibility $p_{i}$ in the input there are up to $n$ successor $p_{o}$ in the output, for $n$ the number of admissible attributes. Each successor $p_{o}$ models a change w.r.t. to an admissible attribute and therefore is a possible resolution of the attribute underspecification. The qualification 'up to' refers to the fact that a possibility is discarded if in its world component the rising event in the possibility does not bring about a change w.r.t. the attribute specified in the possibility. Hence, this update operation combines aspects of both a 'normal' update (information is added to the frame component of a discourse object already on the stack) and a domain expansion operation: it leads to 'branching' in the sense that a possibility in the input information state can have more than one successor. In (10) the definition of successor is given and in (11) the (simplified) interpretation of an attribute and two domain extension operations are supplied.

(10) a. A stack $c^{\prime}$ is a successor of a stack $c, c \preceq c^{\prime}$, iff $c^{\prime}=c^{\sqcap} \alpha$, for some discourse object $\alpha$.

b. A possibility $p^{\prime}=\left\langle c^{\prime}, w^{\prime}\right\rangle$ is an object-successor of possibility $p=\langle c, w\rangle, p \preceq_{o} p^{\prime}$, iff $c^{\prime} \preceq c$ and $w=w^{\prime}$.

c. A possibility $p^{\prime}=\left\langle c^{\prime}, w^{\prime}\right\rangle$ is a frame-successor of possibility $p=\langle c, w\rangle$ w.r.t. position $v_{i}$ and value range restriction $v r, p \preceq_{v_{i}, v r} p^{\prime}$, iff $w=w^{\prime},|c|=\left|c^{\prime}\right|$, $\exists o f_{o} f_{o}^{\prime}$ ATTR. $c[i]=\left\langle o, f_{o}\right\rangle \wedge c^{\prime}[i]=\left\langle o, f_{o}^{\prime}\right\rangle, f_{o} \sqsubseteq f_{o}^{\prime}, \theta\left(f_{o}^{\prime}\right)=\theta\left(f_{o}\right) \cup\{$ ATTR $\}$, $\llbracket v r \rrbracket(\operatorname{range}($ ATTR $))$ and $\forall j .\left(0 \leq j<|c| \wedge j \neq i \rightarrow c[j]=c^{\prime}[j]\right)$.

$$
\begin{array}{ll}
\text { a. } & s[\operatorname{ATTR}(f)]=\left\{p \in s \mid \exists i, j \in \mathbf{N}: p=\langle c, w\rangle, \pi^{2}(c[i])=f, I N\left(\pi^{2}(c[i])\right)=w,\right. \\
& \left.\left\langle\pi^{2}(c[i]), \pi^{1}(c[i]), \pi^{1}(c[j])\right\rangle \in \llbracket \operatorname{ATTR} \rrbracket\right\} . \\
\text { b. } & s[\exists]=\left\{p^{\prime} \mid p \in s \wedge p \preceq_{o} p^{\prime}\right\} . \\
\text { c. } & s\left[\text { update }\left(v_{i}, v r\right)\right]=\left\{p^{\prime} \mid p \preceq_{v_{i}, v r} p^{\prime} \wedge p \in s\right\} .
\end{array}
$$

In (10a), stack $c^{\prime}$ is a successor of $c\left(c \preceq c^{\prime}\right)$ if it extends $c$ by a discourse object. In (10b), a possibility $p^{\prime}$ is an object-successor of $p$ if its stack component is a successor of that of $p$ and the world components are the same. In (10c), a frame-successor $p^{\prime}$ of a possibility $p$ has the same world component as $p$ and its discourse component (stack) extends the frame component of the discourse object at position $v_{i}$ by an attribute ATTR that satisfies the value range restriction $v r$ while leaving all other discourse objects the same. (11) specifies an information state $s$ after update with some information $\phi$. Here, $s[\phi]$ stands for the updated information state. In (11a) $\pi^{k}$ is the $k$-th projection function. $\pi^{1}(c[i])$ is the object stored at position $i$ and $\pi^{2}(c[i])$ its associated frame, i.e. $\pi^{1}(c[i])$ is the root of $\pi^{2}(c[i])$. The interpretation of an attribute tests 
whether the attribute holds between the frame stored at position $i$, the object stored at the same position $i$, and the object stored at position $j$. This operation eliminates possibilities from the information state that do not pass the attribute test. In (11b), $\exists$ non-deterministically extends each possibility in the input by a discourse object. This operation is used, whenever a new indefinite discourse object is introduced. In (11c), update $\left(v_{i}, v r\right)$ expands an information state by adding to the frame component at position $i$ of the discourse component (non-deterministically) an admissible attribute whose value range satisfies the constraint $v r$.

\section{Probabilities: a worked-out example}

The set of attributes satisfying the constraint on the value range in (7) imposed by 'rise' can be quite large. There are two kinds of information by which this set can be constrained: (i) world knowledge of a comprehender and (ii) the preceding or ensuing linguistic context. For example, a comprehender may have knowledge about China's rising economic power so that he expects the sentence 'China rises' to be about this rising economic power. Context adds extra information to frames in form of an update operation on an input frame. For example, the context below, adapted from Cooper (2010), adds the new information to the 'China'-frame that the value of the attribute DIPLOMATIC_ACTIVITY is 'high'. This information can be taken as an indication that 'China rises' is about its political power and not about its economic power.

China's rising will undoubtedly be one of the great dramas of the twenty-first century. China's extraordinary active diplomacy is already transforming East Asia.

In contrast to attributes like TEMPERATURE and PRICE which are excluded for 'China', the above two kinds of information do in general not exclude all other choices. For example, even if a comprehender knows that China's economic power rises and he therefore expects the sentence to be about that attribute, encountering later on the information about the high diplomatic activity may well have the effect that he revises his decision to interpret the sentence as being about China's political power. The information a comprehender has about attributes like TEMPERATURE and PRICE, namely that they are excluded for objects of sort country, is 'hard' information, whereas the kind of information used about the other two attributes above is 'soft' information because it can be revised. We will model soft information in terms of probabilities. $^{11}$

In the rest of this section we will provide a worked-out example which shows how the choice of an attribute can be constrained using a probability distribution which is based on the knowledge of a comprehender who is processing the text in (12).

We begin by defining the probability distribution $\operatorname{Pr}_{s}$ on an information state $s$. Four cases have to be distinguished: (a) an information state with no discourse information (base case), (b) eliminative update, (c) domain extension and (d) expansive update. For cases (b), (c) and (d), $P r_{s}$ needs to be updated. The definitions are given in (13), see also Djalali and Kaufmann (2009) by whom these definitions are inspired.

\footnotetext{
${ }^{11}$ Hard information is then the limiting case where the probability is either 1 (bottom-up information) or 0 (information like TEMPERATURE, which is not defined for countries).
} 
a. $\operatorname{Pr}_{s}(\langle\langle\rangle, w\rangle \mid s):=P(w)$.

b. $\operatorname{Pr}_{s}(p \mid s[\phi]):=\operatorname{Pr}_{s}(p \mid s) / \Sigma_{p^{\prime} \in s[\phi]} \operatorname{Pr}_{s}\left(p^{\prime} \mid s\right)$, if $p \in s[\phi], 0$ otherwise.

c. $\quad \operatorname{Pr}_{s}\left(p^{\prime} \mid s[\exists]\right):=\operatorname{Pr}_{s}(p \mid s) /|D|$, for $p \preceq_{o} p^{\prime}, p \in s, p^{\prime} \in s[\exists]$.

d. $\operatorname{Pr}_{s}\left(p^{\prime} \mid s\left[\right.\right.$ update $\left.\left.\left(v_{i}, v r\right)\right]\right):=\operatorname{Pr}_{s}(p \mid s) /\left|D_{v r}\right|$ with $p=\langle c, w\rangle, c[i]=\left\langle o, f_{o}\right\rangle, p \in s$, $p^{\prime} \in s\left[\right.$ update $\left.\left(v_{i}, v r\right)\right], p \preceq_{v_{i}, v r} p^{\prime}$ and $D_{v r}:=\left\{\right.$ ATTR $\mid \exists f: f_{o} \sqsubseteq_{f} f \wedge$ ATTR $\in \theta(f) \wedge$ $\llbracket v r \rrbracket(\operatorname{range}(\mathrm{ATTR}))\}$.

In an information state with no discourse information, the probabilities are those of the world components (see (13a)). For eliminative updates, the probability is shifted by conditioning, i.e. the new probability of a 'surviving' possibility from the input is got by dividing its probability in the input by the sum of prior probabilities of all surviving possibilities (see (13b)). For the domain extension operation, the probabilities of possibilities in the input are uniformly distributed over their object-successors (see (13c)), whereas in the case of update $\left(v_{i}, v r\right)$ they are uniformly distributed over their frame-successors (see (13d)).

For our example, let us make the following simplifying assumptions. For 'China', only the attributes POLITICAL_POWER and ECONOMIC_POWER satisfy the value range constraint imposed by 'rise'. A discourse object is of the form $\left\langle o, f_{o}\right\rangle$ and not $\left\langle o,\left\langle f_{d}, f_{i}\right\rangle\right\rangle$ since for China both frame components are the same. The initial information state of the comprehender has two possibilities $p_{1}$ and $p_{2}$, both containing no discourse information and with worlds $w_{1}$ and $w_{2}$, respectively. One has $P\left(w_{1}\right)=0.5=P\left(w_{2}\right)$. Applying (13a) yields $\operatorname{Pr}_{s}\left(p_{1} \mid s\right)=0.5=\operatorname{Pr}_{s}\left(p_{2} \mid s\right)$. In $w_{1}$ there is one rising event with China as theme that increases China's political power whereas in $w_{2}$ there is a corresponding rising event that increases China's economic power. Processing (12) starts with introducing China at position 0 (positions in a stack are counted beginning with 0$)$ together with a minimal China-frame. The initial probabilities for $p_{1}$ and $p_{2}$ are not changed, because 'China' has singular reference, being a proper name. Processing 'rising' (or 'rises') introduces a rising event at position 1. Again $P r_{s}$ is not changed since it is assumed that there is one rising event in each world with China as theme. ${ }^{12}$ In addition, for each rising event the China-frame at position 0 in the corresponding possibility is updated with either the attribute POLITICAL_POWER or ECONOMIC_POWER. Hence, each of the two possibilities has two frame-successors $p_{i 1}$ and $p_{i 2}$, yielding an information state $s^{\prime}$ with four possibilities. Applying clause (13d), one gets $\operatorname{Pr}_{s^{\prime}}\left(p_{11} \mid s^{\prime}\right)=\operatorname{Pr}_{s^{\prime}}\left(p_{12} \mid s^{\prime}\right)=\operatorname{Pr}_{s^{\prime}}\left(p_{21} \mid s^{\prime}\right)=\operatorname{Pr}_{s^{\prime}}\left(p_{22} \mid s\right)=0.25$. The probabilities are divided by 2 because there are two attributes satisfying the value constraint. Next it is tested whether a change is effected by the two rising events relative to the two attributes. In $w_{1}$ ECONOMIC_POWER and in $w_{2}$ POLITICAL_POWER fail this test. The corresponding possibilities are discarded. For $P r_{s^{\prime}}$ clause (13b) applies, shifting the probabilities of the two remaining possibilities $p_{11}$ and $p_{22}$ to 0.5 . This is summarized in the table below where we focus on the relevant attributes.

\begin{tabular}{clc} 
world & attribute & $\operatorname{Pr}_{s}$ \\
\hline$p_{11}$ & POLITICAL_POWER & 0.5 \\
$p_{22}$ & ECONOMIC_POWER & 0.5
\end{tabular}

Table 2: Distribution after update with 'China' and 'rising'

\footnotetext{
${ }^{12}$ If there are $n$ objects in the universe, clause (13c) applies first, applying clause (13b) twice, first for the sortal information 'rise' and then to the information that China is the theme, yields the same result as stated in the text.
} 
The discourse information the comprehender has about China in this information state is contained in the frame below.

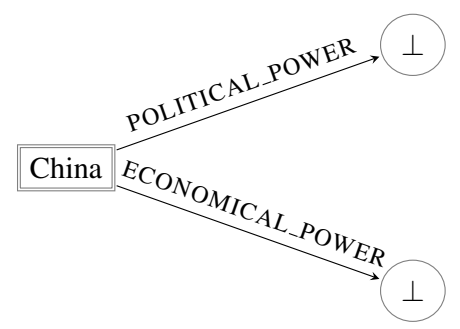

Figure 4: Frame for 'China'

$\perp$ indicates that the comprehender does not know whether a change occurred relative to POLITICAL_POWER or ECONOMIC_POWER. Hence, without further information a comprehender cannot distinguish between the two links marked by '?' in the 'China's rising'-frame below, again representing the frame corresponding to all discourse information in the information state.

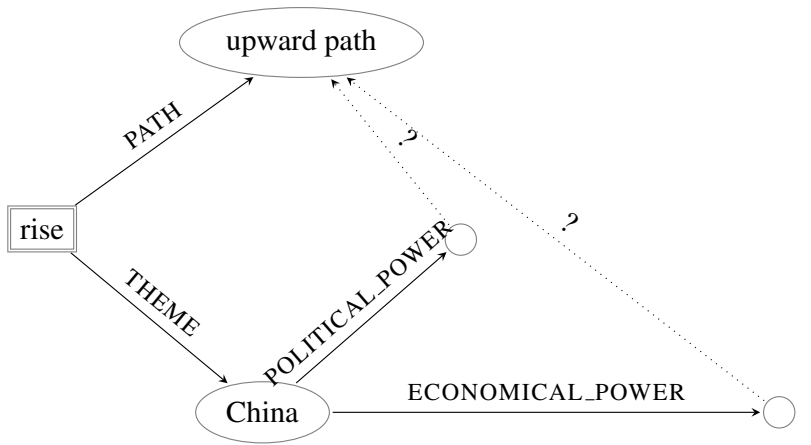

Figure 5: 'China rises'-frame with equal probabilities

In order to account for the contextual information about the attribute DIPLOMATIC_ACTIVITY we have to take the comprehender's expectation about this attribute into account. The (revised) initial distribution is shown below.

\begin{tabular}{llll} 
& attribute & DIPL._ACTIVITY & $\mathrm{P}(\mathrm{w})$ \\
\hline$w_{11}$ & POL._POWER & low & 0.02 \\
$w_{12}$ & POL._POWER & high & 0.48 \\
$w_{21}$ & ECO._POWER & low & 0.25 \\
$w_{22}$ & ECO._POWER & high & 0.25
\end{tabular}

Table 3: Initial distribution with DIPLOMATIC_ACTIVITY $=$ high included

Processing 'China's extraordinary active diplomacy' in (12) updates the (global) 'China'-frame containing the discourse information to that below. 


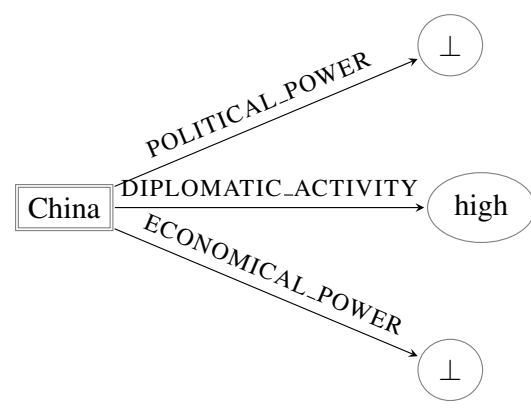

Figure 6: Frame for 'China' extended by contextual knowledge

Updating with this 'hard' information triggers an update in $P r_{s}$ using clause (13b). The new distribution is shown below.

\begin{tabular}{lllc} 
& attribute & DIPL._ACITIVITY & $P r_{s}$ \\
\hline$p_{11}$ & POL._POWER & high & 0.6575 \\
$p_{22}$ & ECO._POWER & high & 0.3425
\end{tabular}

Table 4: 'China rises': distribution after update with DIPLOMATIC_ACTIVITY $=$ high

Hence, using the 'hard' information that China's diplomatic activity is high and the comprehender's expectations about the relation between this kind of activity and the two different powers, results in a probability distribution in which the two attributes no longer have the same probability. Rather, the comprehender has for POLITICAL_POWER a probability of $65.75 \%$. Next, he can apply a decision rule. One of the most simplest ones is 'Choose that possibility with the highest probability'. Applying this rule leads a comprehender to expect that the rise of China in (12) is most likely to be about an increase in its political power. Even if this rule is applied, a change in economic power is still an option. The 'rise'-frame resulting after application of the decision rule is shown below.

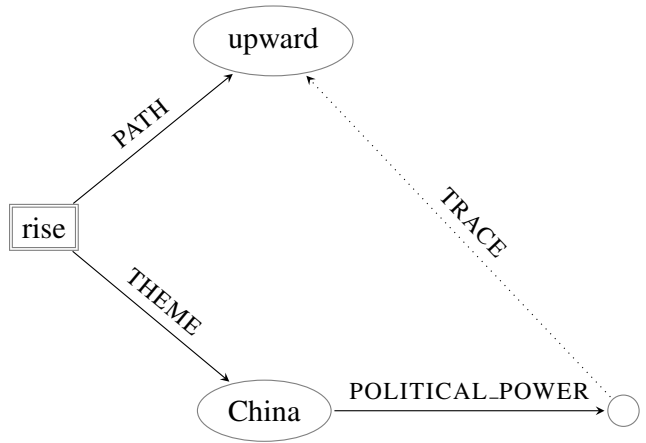

Figure 7: Resolved 'China rises'-frame using probabilities and a decision rule

Thus, if no further information is provided, the comprehender expects that 'China rises' in the context of high diplomatic activity means that the polical power of China is rising.

\section{References}

Blackburn, P. and M. deRijke (1997). Zooming in, zooming out. Journal of Logic, Language and Information 6(1), 5-31. 
Cooper, R. (2010). Frames in formal semantics. In H. Loftsson, E. Rögnvaldsson, and S. Helgadóttir (Eds.), Advances in Natural Language Processing: 7th International Conference on NLP, IceTAL 2010, Reykjavik, Iceland, August 16-18, 2010, pp. 103-114. Berlin, Heidelberg: Springer Berlin Heidelberg.

Djalali, A. and S. Kaufmann (2009). Probabilistic inference in dynamic semantics. In Proceedings of the 10th Symposium on Logic and Language, pp. 99-107. Research Institute for Linguistics, Hungarian Academy of Sciences (HAS), and Theoretical Linguistics Program, Eötvös Lorand University (ELTE).

Fleischhauer, J. and T. Gamerschlag (2014). We are going through changes: How change of state verbs and arguments combine in scale composition. Lingua 141, 30 - 47. SI: Argument Realization in Morphology and Syntax.

Gamerschlag, T., W. Geuder, and W. Petersen (2014). Glück auf der Steiger kommt, a frame account of extensional and intensional 'steigen'. In D. Gerland, C. Horn, A. Latrouite, and A. Ortmann (Eds.), Meaning and grammar of nouns and verbs, pp. 115 - 144. Düsseldorf: Düsseldorf UP.

Kennedy, C. and L. McNally (2005). Scale structure, degree modification, and the semantics of gradable predicates. Language 81(2), 345-381.

Löbner, S. (2011). Concept types and determination. Journal of Semantics 28(3), 279-333.

Löbner, S. (2014). Evidence for frames from human language. In T. Gamerschlag, D. Gerland, R. Osswald, and W. Petersen (Eds.), Frames and Concept Types. Springer.

Naumann, R. (2013). An outline of a dynamic theory of frames. In V. M. G. Bezhanishvili, S. Löbner and F. Richter (Eds.), Proceedings of the 9th International Tbilisi Symposium on Language, Logic and Computation, Volume 7758 of Lecture Notes in Computer Science, pp. 115-137. Springer Berlin Heidelberg.

Naumann, R. and W. Petersen (2017). A formal theory of frames with an application to plural quantification and cross-sentential anaphora. submitted to Journal of Semantics.

Petersen, W. (2007). Representation of concepts as frames. In J. Skilters, F. Toccafondi, and G. Stemberger (Eds.), Complex Cognition and Qualitative Science, Volume 2 of The Baltic International Yearbook of Cognition, Logic and Communication, pp. 151-170.

Petersen, W. and T. Osswald (2014). Concept composition in frames: Focusing on genitive constructions. In T. Gamerschlag, D. Gerland, R. Osswald, and W. Petersen (Eds.), Frames and Concept Types, Volume 94 of Studies in Linguistics and Philosophy, pp. 243-266. Springer.

Pinon, C. (1997). Achievements in an event semantics. In A. Lawson (Ed.), Proceedings SALT VII, Ithaca, NY: Cornell University, pp. 276-293.

Rappaport Hovav, M. and B. Levin (2010). Reflections on manner/result complementarity. In M. Rappaport Hovav, E. Doron, and S. Ivy (Eds.), Lexical Semantics, Syntax and Event Structure, pp. $21-38$. Oxford UP.

van Eijck, J. (2007). Context and the composition of meaning. In H. Bunt and R. Muskens (Eds.), Computing Meaning, pp. 173-193. Dordrecht: Springer Netherlands. 